\title{
De novo truncating variants in the intronless IRF2BPL are responsible for developmental epileptic encephalopathy
}

\author{
F. Tran Mau-Them, MD ${ }^{1,2}$, L. Guibaud, MD, $\mathrm{PhD}^{3,4}$, L. Duplomb, $\mathrm{PhD}^{2}, \mathrm{~B}$. Keren, MD, $\mathrm{PhD}^{5}$, \\ K. Lindstrom, $\mathrm{MD}^{6}$, I. Marey, MD ${ }^{5}$, F. Mochel, MD ${ }^{5,7,8}$, M. J. van den Boogaard, MD, PhD ${ }^{9}$, \\ R. Oegema, MD, PhD ${ }^{9}$, C. Nava, MD, PhD ${ }^{5}, A$. Masurel, $\mathrm{MD}^{10}, \mathrm{~T}$. Jouan, $\mathrm{BS}^{1,2}, \mathrm{~F}$. E. Jansen, $\mathrm{MD}^{11}$, \\ M. Au, MBE, MS ${ }^{12}$, Agnes H. Chen, MD ${ }^{13}$, M. Cho, ScM, CGC ${ }^{14}$, Y. Duffourd, MsC ${ }^{2}$, E. Lozier, MS ${ }^{15}$ \\ F. Konovalov, PhD ${ }^{15}$, A. Sharkov, MD ${ }^{15,16}$, S. Korostelev, MD ${ }^{15}$, B. Urteaga, MsC ${ }^{2}$, P. Dickson, MD ${ }^{17}$, \\ M. Vera, MD, PhD ${ }^{17}$, Julián A. Martínez-Ágosto, MD, PhD ${ }^{20}$, A. Begemann, MD ${ }^{18}$, M. Zweier, PhD ${ }^{18}$, \\ T. Schmitt-Mechelke, MD ${ }^{19}$, A. Rauch, MD ${ }^{18}$, C. Philippe, PhD ${ }^{1,2}$, K. van Gassen, PhD ${ }^{9}$, S. Nelson, MD ${ }^{20}$, \\ J. M. GrahamJr, MD, ScD ${ }^{12}$, J. Friedman, $\mathrm{MD}^{21}$, L. Faivre, MD, PhD ${ }^{2,10}$, H. J. Lin, $\mathrm{MD}^{17}$, \\ C. Thauvin-Robinet, $\mathrm{MD}, \mathrm{PhD}^{1,10}$ and A. Vitobello, $\mathrm{PhD}^{1,2}$
}

Purpose: Developmental and epileptic encephalopathies (DEEs) are severe clinical conditions characterized by stagnation or decline of cognitive and behavioral abilities preceded, accompanied or followed by seizures. Because DEEs are clinically and genetically heterogeneous, next-generation sequencing, especially exome sequencing (ES), is becoming a first-tier strategy to identify the molecular etiologies of these disorders.

Methods: We combined ES analysis and international data sharing.

Results: We identified 11 unrelated individuals with DEE and de novo heterozygous truncating variants in the interferon regulatory factor 2-binding protein-like gene (IRF2BPL). The 11 individuals allowed for delineation of a consistent neurodevelopmental disorder characterized by mostly normal initial psychomotor development followed by severe global neurological regression and epilepsy with nonspecific electroencephalogram (EEG) abnormalities and variable central nervous system (CNS) anomalies. IRF2BPL, also known as enhanced at puberty protein 1 (EAP1), encodes a transcriptional regulator containing a $\mathrm{C}$ terminal RING-finger domain common to E3 ubiquitin ligases. This domain is required for its repressive and transactivating transcriptional properties. The variants identified are expected to encode a protein lacking the C-terminal RING-finger domain.

Conclusions: These data support the causative role of truncating $I R F 2 B P L$ variants in pediatric neurodegeneration and expand the spectrum of transcriptional regulators identified as molecular factors implicated in genetic developmental and epileptic encephalopathies.

Genetics in Medicine (2019) 21:1008-1014; https://doi.org/10.1038/s41436018-0143-0

Keywords: developmental epileptic encephalopathies; exome sequencing; data sharing; IRF2BPL

\section{INTRODUCTION}

Epilepsy and severe intellectual disability (ID) are frequent comorbid conditions, with epilepsy affecting more than half of individuals with severe ID. ${ }^{1}$ Two different patterns may occur. Either the seizures precede cognitive impairment or regression in the epileptic encephalopathies (EEs), ${ }^{2,3}$ or, for developmental encephalopathies (DEs), epilepsy develops on a background of developmental delay or neurologic regression. ${ }^{2,3}$ Patients with apparently normal development can lose previously acquired skills (e.g., Rett syndrome [MIM 312750] or in inborn errors of metabolism such as FOLR1 deficiency causing severe leukoencephalopathy ${ }^{4}$ ) or patients with a background of developmental delay can worsen (i.e., Angelman syndrome [AS; MIM 105830]). Distinguishing between these two patterns is difficult, and the term developmental and epileptic encephalopathy (DEE) has emerged as a result, defining a group of conditions characterized by the cooccurrence of epilepsy and intellectual disability (ID), typically with developmental plateau or regression associated with frequent epileptiform activity. ${ }^{2,3}$ DEEs encompass a wide range of etiologies, including both acquired and genetic causes resulting in a clinically and genetically heterogeneous group of rare or ultrarare diseases. To date, more than 500 different genes have been associated with an increased risk of epilepsy

Correspondence: F Tran Mau-Them (frederic.tran-mau-them@u-bourgogne.fr) or A Vitobello (antonio.vitobello@u-bourgogne.fr). ${ }^{\#}$ Affiliations are listed at the end of the paper. These authors contributed equally: F. Tran Mau-Them, C. Thauvin-Robinet, A. Vitobello. 
or seizures. ${ }^{5}$ These involve multiple pathways and functions, including metabolite and ion transport, transcription regulation, DNA repair processes, myelination, and peroxisomal function. ${ }^{2}$ Nevertheless, most DEEs remain unexplained at the molecular level despite chromosomal microarray analysis and epilepsy gene panel testing, suggesting that further DEE genes have yet to be discovered. In this context, exome and genome sequencing (ES/GS) have demonstrated efficiency in the identification of new causal genes ${ }^{6,7}$ by uncovering an increased burden of de novo variants. ${ }^{1}$ Indeed, sporadic lossof-function (LoF) variants were initially and frequently implicated in different neurodevelopmental disorders such as ID, autism spectrum disorder, and schizophrenia. ${ }^{8}$ More recently, exome and genome sequencing analyses on cohorts with large sample size have identified spatial clustering of de novo missense variants in candidate neurodevelopmental disorder-associated genes and recurrent missense variants in DEEs. ${ }^{9}$ The findings are compatible with functional impairment of specific domains (dominant-negative or gain-offunction effects), rather than haploinsuffiency, as a causal mechanism for some genes. We report a novel molecular cause of DEE with regression caused by a sporadic truncating de novo variant in IRF2BPL.

\section{MATERIALS AND METHODS}

By using trio-based ES analysis (Methods provided in the Supplemental data), we identified a de novo heterozygous truncating variant (chr14:g.77493617G>C - NM_024496.3: c.519C $>$ G) in IRF2BPL (MIM 611720) in one affected boy. Array comparative genomic hybridization (array CGH) and ES analyses did not identify de novo copy-number or other single-nucleotide variants accounting for his clinical presentation. He had normal early psychomotor development followed by lethal progressive neurological regression and epilepsy (patient 1; Table 1 and Supplemental data). Sanger sequencing in the patient and his parents confirmed de novo occurrence of the truncating IRF2BPL variant $\left(\mathrm{p} \cdot T y r 173^{*}\right)$. This variant was absent from the gnomAD database (see Online databases). We used data sharing through GeneMatcher, ${ }^{10}$ GeneDx (see Online databases), and private networking to identify similar cases. Each group contributed to phenotype information from their center, in accordance with local institutional Review Board policies.

Further methods can be found in the Supplemental Data.

\section{RESULTS}

We ascertained ten additional individuals with overlapping phenotypes and de novo heterozygous truncating IRF2BPL variants, also identified by ES (research or clinical; Table 1, Fig. 1b). IRF2BPL is highly intolerant to LoF according to ExAC with a probability of LoF intolerance (pLI) of 0.97 . Although some LoF variants are reported in the ExAC/ gnomAD cohorts, most of them appear to be dubious calls. In addition IRF2BPL is also predicted to have a low likelihood of exhibiting haploinsufficiency (DECIPHER's haploinsufficiency score $\% \mathrm{HI} 48.87 \%$; see Online databases). Accordingly, RNA analyses on patient-derived fibroblasts are in favor of nonsense-mediated decay (NMD) escape because the mutated allele is expressed with a ratio of at least 1:1 with the wild-type (WT) allele, suggesting that the shorter protein might be translated (Fig. 1c). The 11 truncating variants included 6 nonsense and 5 frameshift variants, all absent from the gnomAD database (see Online databases) (Table 1, Fig. 1b). Detailed retrospective phenotyping of the 11 individuals delineated a consistent neurodevelopmental disorder characterized by secondary global neurological regression usually starting early in childhood, frequently with seizures (Table 1). Seven of the 11 cases presented a similar course of development, with initially normal motor development and only mild speech delay. Two had mild motor developmental delay and mild speech delay. These nine patients later displayed neurological regression with typical onset mostly before age 7 years (6/9 cases; range: 1 to 17 years, mean: 6 years). Among the nine individuals, two (patients 3 and 4) presented with late-onset neurological regression after ages 10 and 17 years (respectively) associated with myoclonus, and less severe outcomes seeing as both were still walking at age 23 years. The patient without regression was only 3 years and 7 months old at the latest examination and therefore regression may not yet have been apparent (patient 10). Finally, two children (patients 7 and 11) presented at 2.5 and 3.5 months old with a more severe phenotype that included clusters of asymmetric tonic spasms and clonic seizures with a hypsarrhythmia-like pattern on the EEG, leading to severe EE with quadriplegic hypotonic-ataxic cerebral palsy. Patients with early-onset regression seem to have a more severe outcome.

Epilepsy was frequent (7/11 cases), starting from the age of 6 months to 26 years. There were variable manifestations including infantile spasms, myoclonus, and tonic or clonic seizures with nonspecific EEG pattern anomalies (Table 1). Epilepsy was severe, usually with intractable seizures. Only $2 / 8$ patients were responsive to treatment with clonazepam and at least one other medication (patients 1 and 4). Additional neurological features varied from severe tetraparesis (3/11 cases) to a cerebellar syndrome with ataxia (3/ 11 cases), dysarthria (2/11 cases), and nystagmus (2/11 cases). Other abnormalities included hypotonia (5/11 cases), dystonia (3/11 cases), dysphagia (3/11 cases), and infrequent microcephaly ( $1 / 11$ cases) (Table 1$)$. Brain MRI was normal in $4 / 10$ cases but showed variable CNS anomalies in $6 / 10$ cases, including diffuse or focal brain or cerebellar atrophy (6/6 cases) (Fig. 1a, Table 1). Muscle biopsy (4/11 cases) identified several nonspecific anomalies in $3 / 4$ cases including T2 fiber atrophy, negative cytochrome $c$ oxidase fibers, nemalin rods, or mild variation in fiber size with rare ragged red-like fibers. Muscle electron transport chain studies performed in patient 1 showed mild generalized deficiencies that could indicate primary or secondary mitochondrial defects. 

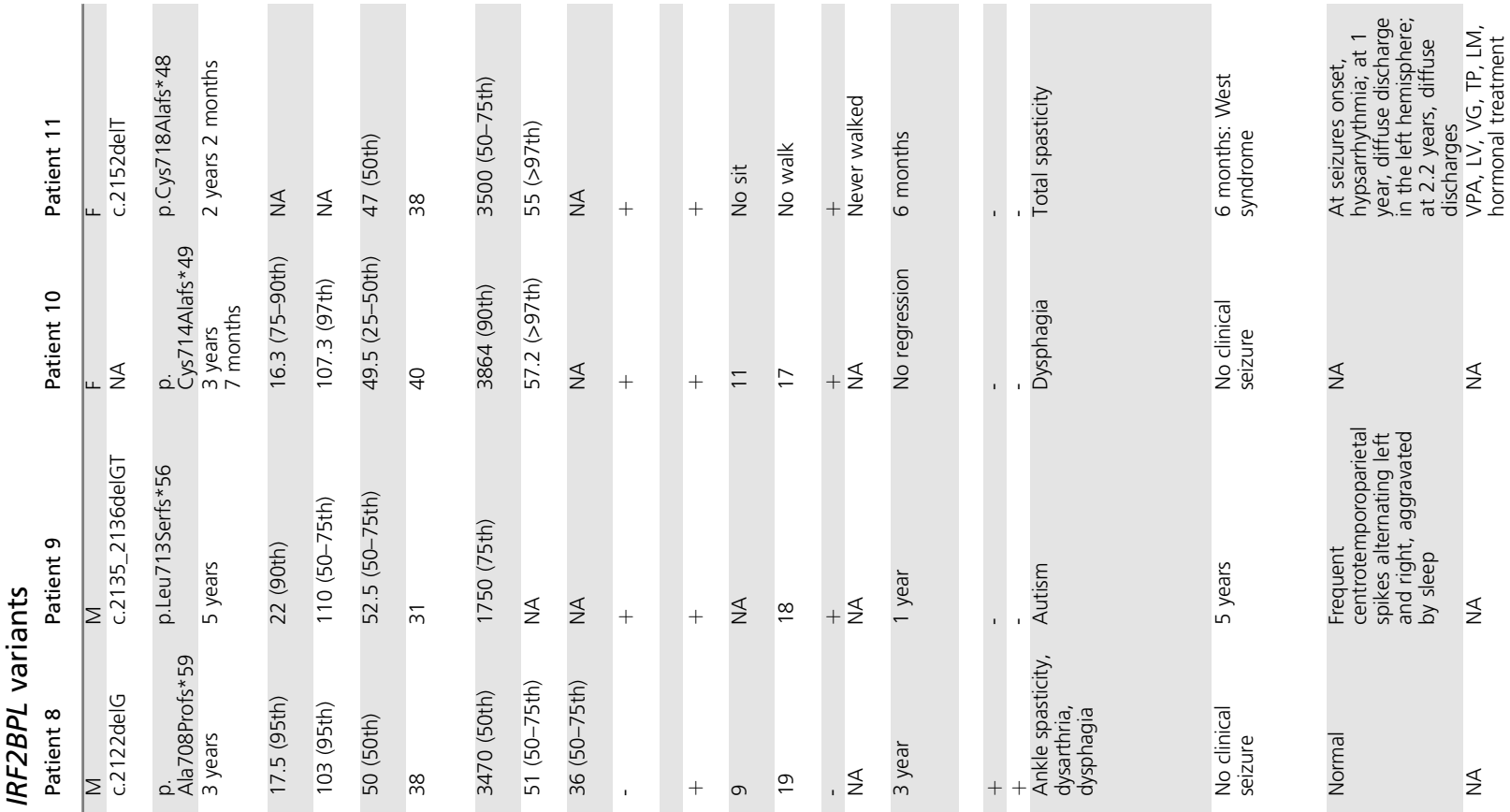

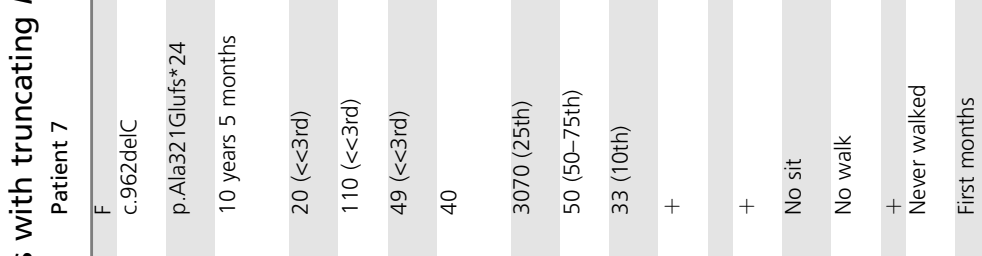

艺

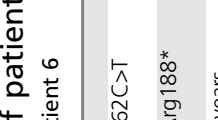

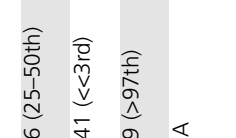

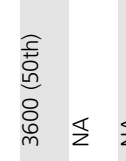
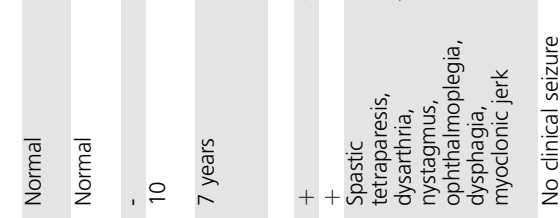

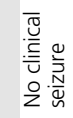

$\frac{\overline{\widetilde{g}}}{\overline{\underline{g}}}$

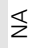

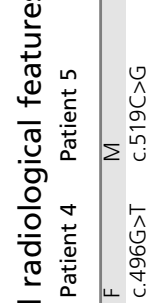

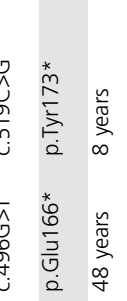

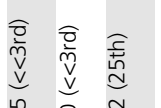

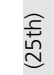

$\sqrt{2}$

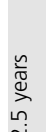

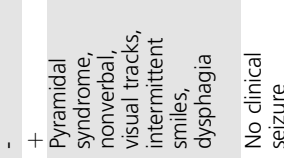

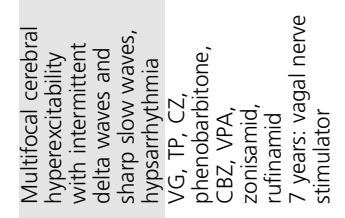

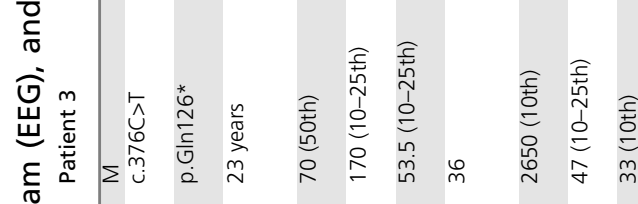

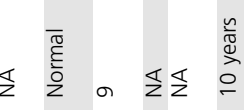

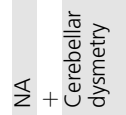

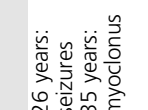

ㄴ. 땠 है है

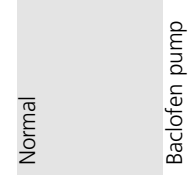

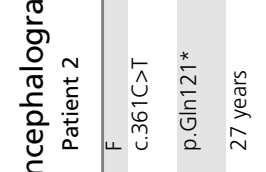

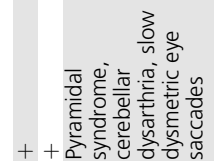

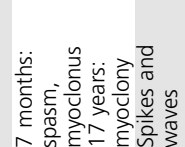

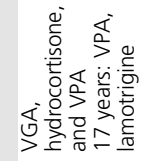

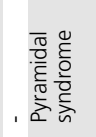

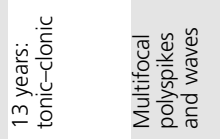

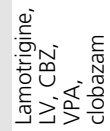

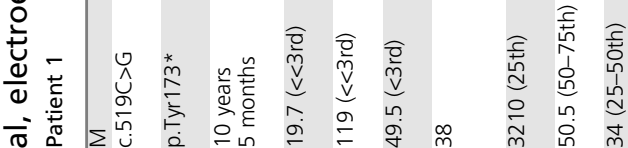

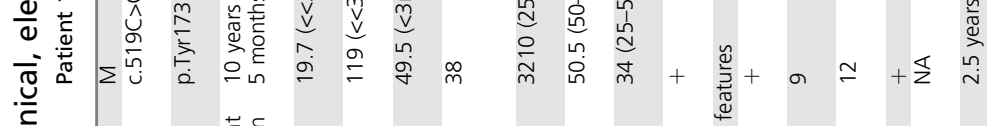

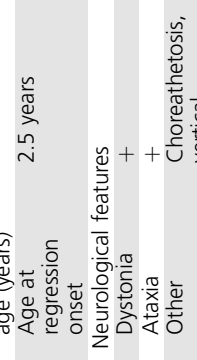

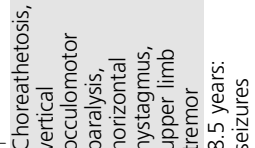

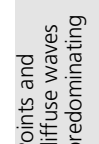

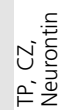

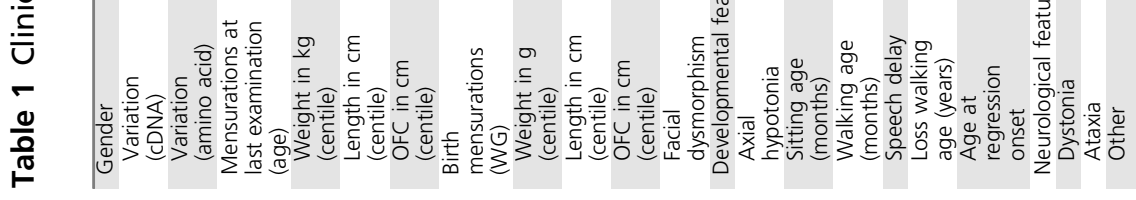




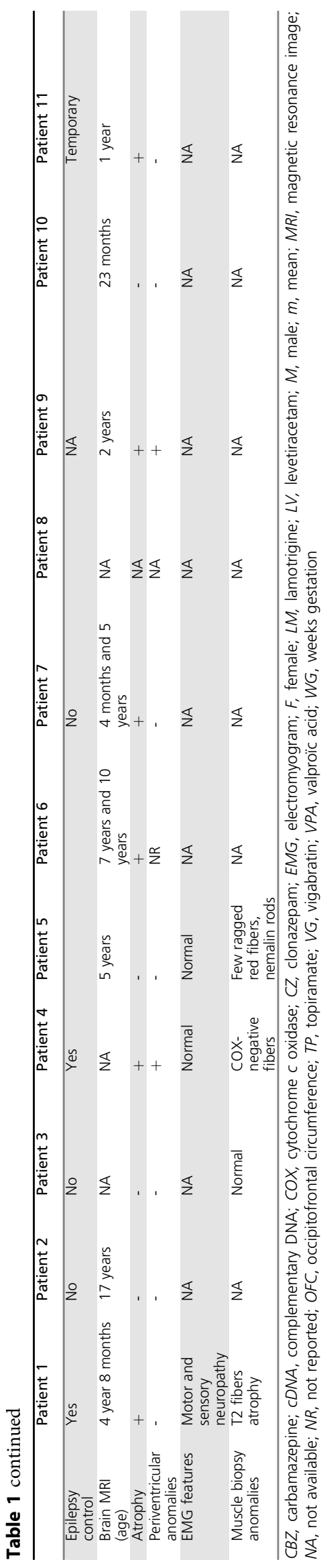

\section{DISCUSSION}

The occurrence of variants in IRF2BPL in 11 unrelated individuals with similar phenotypes strongly implicates de novo IRF2BPL truncating variants in DEE, characterized by progressive encephalopathy, which typically appears after mostly normal initial development, and frequently with epilepsy. Patients usually present with severe epilepsy and intractable seizures. Establishing a correlation between the degree of seizure control and later neurologic/cognitive outcomes was not feasible because of the difficulties associated with systematic IQ assessment. Interestingly, patients with early-onset regression displayed a more severe outcome. A similar phenotype may occur in other genetic syndromes (i.e., Rett syndrome or neurometabolic encephalopathies such as adrenoleukodystrophy [ALD; MIM 300100] or mitochondrial leukoencephalopathies). Rett syndrome is caused by heterozygous LoF pathogenic variants in $M E C P 2$, a regulator of chromatin compaction and gene transcription. Although the normal initial development and the mean age at the onset of regression (6 years) in IRF2BPL patients are similar to Rett syndrome, some cardinal features are absent, namely stereotypic hand movements and acquired microcephaly. Despite overlapping clinical features with Angelman syndrome (AS), such as normal initial development followed by severe delay, other features such as the movement disorder (ataxia), epilepsy, microcephaly, and characteristic behavior (inappropriate happy demeanor) are lacking. In addition, the absence of significant white matter anomalies on brain MRIs of the reported patients is incompatible with leukoencephalopathies (Fig. 1a). However, in one case, muscle electron transport chain studies potentially suggested primary or secondary mitochondrial dysfunction. ${ }^{11}$ Array CGH and ES analyses did not identify further de novo copy-number or other single-nucleotide variants accounting for the variable age of onset and degree of severity of the clinical phenotype.

The ExAC pLI score of IRF2BPL (interferon regulatory factor 2-binding protein-like) indicates high LoF intolerance. It belongs to a group of human intronless genes that are known to possibly escape nonsense-mediated decay (NMD), despite presence of a premature stop codon. ${ }^{12}$ This hypothesis was confirmed by RNA analysis on patient-derived fibroblasts. Interestingly, two copy-number variants partially overlapping IRF2BPL have been reported in the general population (Database of Genomic Variants; DGV): a copynumber loss of $31.19 \mathrm{~kb}$ (DGV nsv565210) and an unknown structural variant (DGV nsv510384) whose impact on $I R F 2 B P L$ transcription and expression pattern are unknown. The DECIPHER database (see Online databases) reports eight cases composed of four copy-number gains, one duplication, and three deletions (spanning $184.92 \mathrm{~kb}$ to $30.16 \mathrm{Mb}$ ) of unknown or uncertain pathogenicity. Among the patients showing de novo deletions (ID 265207 and 277160) or duplication (ID 253152) no phenotypic features were available. Among the patients with unknown inheritance of the variant, intellectual disability was a common feature when this information was available. One patient carried a $1.39-\mathrm{Mb}$ 
a
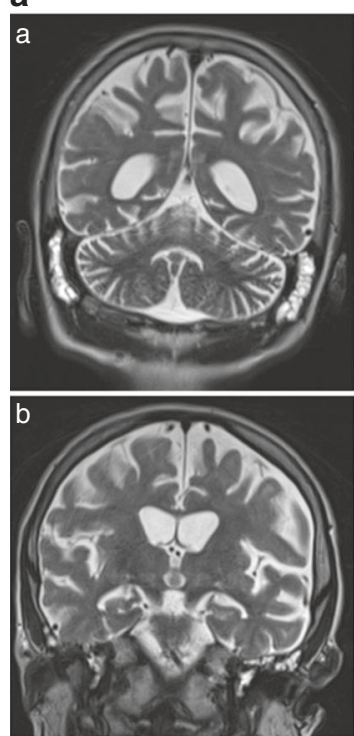

b
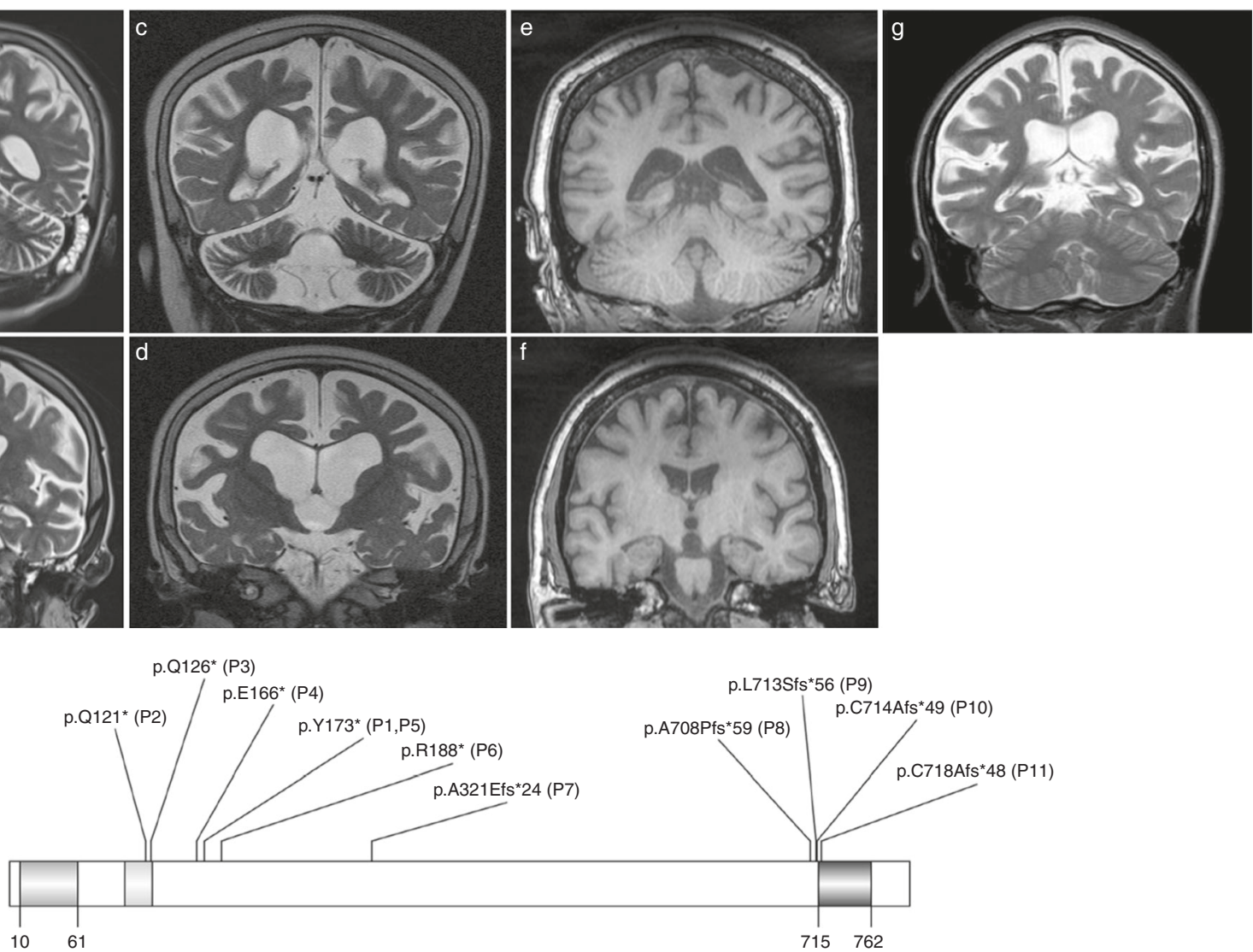

c

P3 C. $376 \mathrm{C}>\mathrm{T}$
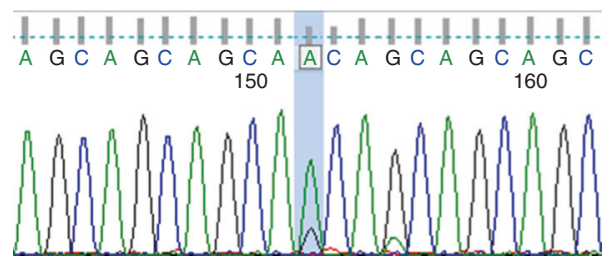

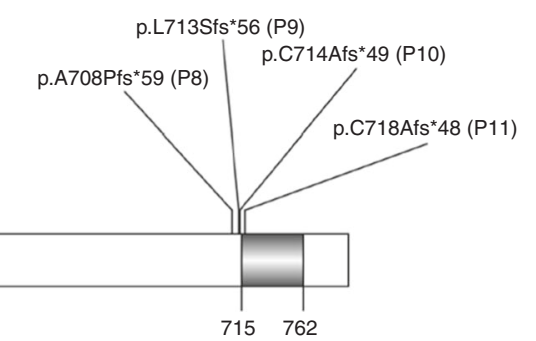

P7 c.962delC

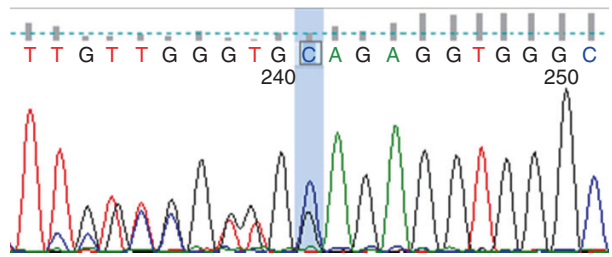

Fig. 1 Medical imaging and molecular features from patients with IRF2BPL truncating variants. a Brain magnetic resonance image (MRI) coronal sections (T1 \& T2 sequences) from affected patients $(\mathbf{a}, \mathbf{b}) 1,(\mathbf{c}, \mathbf{d}) 4,(\mathbf{e}, \mathbf{f}) 7$, and $\mathbf{g} 6$ showing nonspecific discrete to severe parenchymal rarefaction involving both supra and infratentorial structures associated to a vacuo ventricular dilatation without changes in white matter intensity. b Protein structure of IRF2BPL showing positions of reported variants from reported patients (obtained with DOG 2.0; see Online databases). c Reverse transcription polymerase chain reaction (PCR) electrophorograms from patients 3 and 7 derived fibroblast showing presence of the variation.

deletion also present in the father. Regarding haploinsufficiency in mammals, rodents subjected to hypothalamic targeted short hairpin RNA (shRNA) experiments displayed anomalies in puberty and hormonal cycling. ${ }^{13}$ Overall, these tissue-specific IRF2BPL downregulation experiments do not allow us to infer the effects of a haploinsufficiency in humans. Moreover, no endocrine phenotype has been described in our cohort of patients. Thus, although rodent experiments suggest that haploinsufficiency of IRF2BPL may lead to an endocrine phenotype, this mechanism appears unlikely in our patients. Instead, our patients likely have a truncated protein. Also, at least one deletion carrier from DECIPHER was healthy enough to father a child, which would exclude DEE.

IRF2BPL (formerly known as EAP1; enhanced at puberty 1) encodes a nuclear, 796-amino acid protein composed of an N- terminal zinc-finger domain (positions 10-61), a polyglutamine region (polyQ; positions 103-127) and a C-terminal RING-finger domain (really interesting new gene; C3HC4type; positions 715-762), with the latter usually found in members of the E3 ligases family. ${ }^{14}$ The C3HC4 RING-finger takes part in ubiquitinating substrates targeted for degradation. ${ }^{15,16}$ The ubiquitin-proteasome system (UPS) has been described in the nucleus where IRBF2BPL proteins are localized. ${ }^{15}$ Interestingly, all of the truncating IRBF2BPL variants are expected to impact the C-terminal RING-finger of the IRF2BPL protein (Fig. 1b). It is also noteworthy that the deregulation of $\mathrm{E} 3 \mathrm{Ub}$ ligases or accumulation of undegraded ubiquinated protein aggregates have been put forward as major causes of neurodegenerative disorders or human $\mathrm{EE}^{16}$ such as AS (caused by pathogenic variants in UBE3A [MIM 
601623]). Interestingly, in ALD, the accumulation of toxic undegraded very long chain fatty acids-dependent reactive oxygen species inhibits the UPS and autophagy, leading to neuronal degeneration. ${ }^{17}$ C-terminal RING-finger integrity is also required for IRF2BPL autoregulation because IRF $2 B P L$ represses its own transcription. ${ }^{18}$ Our results in fibroblasts argue against this because no quantitative abnormality was observed. Moreover, deletion of the RING-finger abolished $I R F 2 B P L$ transcriptional repression in neuronal (GT1-7) and nonneuronal (GripTite 293 MSR) cell lines leading to unregulated IRF2BPL transcription. ${ }^{18}$

$I R F 2 B P L$ transcripts are ubiquitously expressed in human tissues, including the brain (see Online databases; GTEx Portal). Though the function and role of IRF2BPL during brain development are poorly understood, it has been previously identified as a dual transcriptional regulator of neuronal networks in the female reproductive axis of rodent and nonhuman primates. ${ }^{13}$ The IRF2BPL RING-finger is also required for its transcriptional function. This C3HC4 RINGfinger domain shares high homology with the RING-finger of the IRF2BP2 protein. Removal of this domain in IRF2BP2 leads to defective binding with CBFA2T3 (CBFA2/RUNX1 translocation partner 3; also known as ETO2) and to impaired gene regulation. ${ }^{19}$ IRF2BPL also interacts with a transcriptional complex (DIF-1) involved in apoptosis regulation in breast cancer through repression of the FASTKD2 proapoptotic gene (MIM 612322). ${ }^{20}$ This multiprotein complex forms through protein-protein interactions involving the IR2BPL Zn-finger domain. Knockdown of IRF2BPL in 293T cells disrupts the stability of the complex. ${ }^{20}$ These protein interactions are also decreased in IRF2BPL Zn-finger mutated $\mathrm{HeLa}$ cells but remain normal in C3HC4 RING-finger mutated cells. ${ }^{20}$ Interestingly, all of the truncating IRF2BPL variants are predicted to conserve the $\mathrm{Zn}$-finger domain. Thus, these variants may not destabilize the DIF-1 complex, and may not lead to increased FASTKD2 expression as described above. Overall, IRF2BPL appears to be a transcription regulator that interacts with multiple protein complexes and regulates transcriptional networks. Remarkably, transcription regulation disturbances have been implicated as a major mechanism of encephalopathies such as Rett syndrome.

In conclusion, we found de novo truncating variants in $I R F 2 B P L$ in 11 unrelated individuals with similar DEE. This discovery strongly suggests the role of these variants in a phenotype characterized by typical initial normal psychomotor development followed by progressive neurological regression and epilepsy.

\section{Online databases}

GeneDx: https://www.genedx.com/, GeneMatcher: https:// www.genematcher.org/, Genome Aggregation Database: http://gnomad.broadinstitute.org/, GTEx Portal: http://www. gtexportal.org/, PubMed: https://www.ncbi.nlm.nih.gov/ pubmed/, University of California-Santa Cruz (UCSC) Genome Browser: https://www.genome.ucsc.edu/, DOG 2.0: http://dog.biocuckoo.org/

\section{ELECTRONIC SUPPLEMENTARY MATERIAL}

The online version of this article (https://doi.org/10.1038/s41436018-0143-0) contains supplementary material, which is available to authorized users.

\section{ACKNOWLEDGEMENTS}

We thank the patients and their families for their participation. We thank the Integragen Society and CNRGH (Centre National de Recherche en Génétique Humaine) for exome analysis in some cases. This work was supported by grants from the Regional Council of Burgundy (PARI) and the FEDER. We also thank the National Heart, Lung, and Blood Institute (NHLBI) GO Exome Sequencing Project and its ongoing studies, which produced and provided exome variant calls for comparison: the Lung GO Sequencing Project (HL-102923), the WHI Sequencing Project ( $\mathrm{HL}-102924)$, the Broad GO Sequencing Project (HL-102925), the Seattle GO Sequencing Project (HL-102926), the Heart GO Sequencing Project (HL-103010), and the Rare Disease Initiative Zürich (RADIZ), Clinical Research Priority Program for Rare Diseases of the University of Zurich.

\section{CONFLICT OF INTEREST}

The authors declare no conflicts of interest.

\section{REFERENCES}

1. Hamdan FF, Myers CT, Cossette $P$, et al. High rate of recurrent de novo mutations in developmental and epileptic encephalopathies. Am J Hum Genet. 2017;101:664-685.

2. McTague A, Howell KB, Cross JH, Kurian MA, Scheffer IE. The genetic landscape of the epileptic encephalopathies of infancy and childhood. Lancet Neurol. 2016;15:304-316.

3. Scheffer IE, Berkovic S, Capovilla G, et al. ILAE classification of the epilepsies: position paper of the ILAE Commission for Classification and Terminology. Epilepsia. 2017;58:512-521.

4. Kobayashi Y, Tohyama J, Akiyama T, Magara S, Kawashima H, Akasaka $N$, et al. Severe leukoencephalopathy with cortical involvement and peripheral neuropathy due to FOLR1 deficiency. Brain Dev. 2017;39:266-270.

5. Weber YG, Biskup S, Helbig KL, Von Spiczak S, Lerche $H$. The role of genetic testing in epilepsy diagnosis and management. Expert Rev Mol Diagn. 2017;17:739-750.

6. Trump N, McTague A, Brittain $H$, et al. Improving diagnosis and broadening the phenotypes in early-onset seizure and severe developmental delay disorders through gene panel analysis. J Med Genet. 2016:53:310-317.

7. Hardies K, Weckhuysen S, De Jonghe P, Suls A. Lessons learned from gene identification studies in Mendelian epilepsy disorders. Eur J Hum Genet. 2016;24:961-967.

8. Deciphering Developmental Disorders Study. Prevalence and architecture of de novo mutations in developmental disorders. Nature. 2017;542:433-438.

9. Lelieveld $\mathrm{SH}$, Wiel L, Venselaar H, Pfundt R, Vriend G, Veltman JA, et al. Spatial clustering of de novo missense mutations identifies candidate neurodevelopmental disorder-associated genes. Am J Hum Genet. 2017; 101:478-484

10. Sobreira N, Schiettecatte F, Valle D, Hamosh A. GeneMatcher: a matching tool for connecting investigators with an interest in the same gene. Hum Mutat. 2015;36:928-930.

11. Niyazov DM, Kahler SG, Frye RE. Primary mitochondrial disease and secondary mitochondrial dysfunction: importance of distinction for diagnosis and treatment. Mol Syndromol. 2016;7:122-137.

12. Brocke KS, Neu-Yilik G, Gehring NH, Hentze MW, Kulozik AE. The human intronless melanocortin 4-receptor gene is NMD insensitive. Hum Mol Genet. 2002;11:331-335. 
13. Dissen GA, Lomniczi A, Heger S, Neff TL, Ojeda SR. Hypothalamic EAP1 (enhanced at puberty 1 ) is required for menstrual cyclicity in nonhuman primates. Endocrinology. 2012;153:350-361.

14. Budhidarmo R, Nakatani Y, Day CL. RINGs hold the key to ubiquitin transfer. Trends Biochem Sci. 2012;37:58-65.

15. von Mikecz $A$, Chen $M$, Rockel $T$, Scharf $A$. The nuclear ubiquitinproteasome system: visualization of proteasomes, protein aggregates, and proteolysis in the cell nucleus. Methods Mol Biol. 2008;463:191-202.

16. Zheng Q, Huang T, Zhang L, Zhou Y, Luo H, Xu H, et al. Dysregulation of ubiquitin-proteasome system in neurodegenerative diseases. Front Aging Neurosci. 2016;8:303

17. Fourcade S, Ferrer I, Pujol A. Oxidative stress, mitochondrial and proteostasis malfunction in adrenoleukodystrophy: a paradigm for axonal degeneration. Free Radic Biol Med. 2015;88:18-29.
18. Mueller JK, Koch I, Lomniczi A, et al. Transcription of the human EAP1 gene is regulated by upstream components of a puberty-controlling tumor suppressor gene network. Mol Cell Endocrinol. 2012;351:184-198.

19. Stadhouders R, Cico A, Stephen T, et al. Control of developmentally primed erythroid genes by combinatorial co-repressor actions. Nat Commun. 2015;6:8893.

20. Yeung KT, Das S, Zhang J, Lomniczi A, Ojeda SR, Xu CF, et al. A novel transcription complex that selectively modulates apoptosis of breast cancer cells through regulation of FASTKD2. Mol Cell Biol. 2011;31:2287-2298.

${ }^{1}$ UF Innovation en diagnostic genomique des maladies rares, CHU Dijon Bourgogne, Dijon, France. ${ }^{2}$ INSERM UMR1231 GAD, F21000 Dijon, France. ${ }^{3}$ Universite Claude Bernard Lyon I, CHU de Lyon, Lyon, France. ${ }^{4}$ Service de Radiologie, Hopital-Femme-MèreEnfant, Hospices Civils de Lyon, Lyon, France. ${ }^{5}$ Departement de Genetique, Hopital Pitie-Salpetriere, Paris, France. ${ }^{6}$ Division of Genetics and Metabolic Phoenix Children's Hospital, Phoenix, Arizona, USA. ${ }^{7}$ Inserm U 1127, CNRS UMR 7225, Sorbonne Universites, UPMC Univ Paris 06 UMR S 1127, Institut du Cerveau et de la Moelle epinière, ICM, Paris, France. ${ }^{8}$ Reference Center for Adult Neurometabolic Diseases, Pitie-Salpêtrière University Hospital, Paris, France. ${ }^{9}$ Department of Genetics, University Medical Center, Utrecht, The Netherlands. ${ }^{10}$ Centre de Reference maladies rares « Anomalies du Developpement et syndrome malformatifs " de I'Est, Centre de Genetique, Hopital d'Enfants, FHU TRANSLAD, CHU Dijon Bourgogne, Dijon, France. ${ }^{11}$ Department of Child Neurology, Brain Center Rudolf Magnus, University Medical Center, Utrecht, The Netherlands. ${ }^{2}$ Department of Pediatrics, Division of Medical Genetics, Cedars-Sinai Medical Center and Harbor-UCLA Medical Center, Los Angeles, California, USA. ${ }^{13}$ Division of Pediatric Neurology, Department of Pediatrics, Harbor-UCLA Medical Center, Los Angeles, California, USA. ${ }^{14}$ GeneDx, Gaithersburg, Maryland, USA. ${ }^{15}$ Genomed Ltd., Moscow, Russia. ${ }^{16}$ Veltischev Research and Clinical Institute for Pediatrics of the Pirogov Russian National Research Medical University, Moscow, Russia. ${ }^{17}$ Division of Medical Genetics, Department of Pediatrics, Harbor-UCLA Medical Center, Torrance, California, USA. ${ }^{18}$ Institute of Medical Genetics, University of Zurich, Schlieren, Zurich, Switzerland. ${ }^{19}$ Division of Pediatric Neurology, Children's Hospital, Lucerne, Switzerland. ${ }^{20}$ Departments of Human Genetics and Psychiatry, David Geffen School of Medicine at UCLA, Los Angeles, California, USA. ${ }^{21}$ Department of Neurosciences and Pediatrics UCSD/Rady Children's Hospital San Diego, Rady Children's Institute for Genomic Medicine, San Diego, California, USA 